\title{
EL ESTADO Y SU DERECHO, LA COMUNIDAD Y SUS INSTITUCIONES: UN CASO DEL PERÚ, LA RONDA CAMPESINA
}

\section{The state and its legal system, the community with its institutions: a case in Peru, the ronda campesina}

\section{LEIF KORSBAEK*}

\section{Fecha de recepción: 28 de junio de 2016- Fecha de aprobación: 28 de noviembre de 2016}

\section{Resumen}

En este trabajo se presenta información acerca de la institución legal comunitaria de "la ronda campesina", su trayectoria histórica y sus conflictos con el aparato legal del Estado. Se acerca a una primera comparación doble: se compara la región andina central con su ronda campesina con la región de Mesoamérica y su institución predilecta, el sistema de cargos, y se plantea hipotéticamente que la ronda campesina (de la cual están surgiendo réplicas en México, en Mesoamérica) ha surgido históricamente del sistema de cargos (que existe también en la región andina, menos estudiado pero conocido bajo el nombre de "el sistema de fiestas") bajo la presión de la política neoliberal. Luego se realiza una reflexión acerca del trabajo de campo y la etnografía, y de la importancia de los métodos y técnicas de la Escuela de Manchester, resumiendo en la conclusión algunos de los resultados de la investigación de la ronda campesina y planteando una segunda etapa de esta investigación, que invoca una comparación con la región de Mesoamérica y el sistema de cargos.

Palabras clave: la ronda campesina, el sistema de cargos, la región andina central, Mesoamérica, etnografía del Estado

\begin{abstract}
Information is offered about the legal community institution known as the ronda campesina, its historical career and its confrontations with the state and its legal system. A first approach is made toward a double comparison: the Andean region, with her ronda campesina (of which replicas are popping up in Mexico, in Mesoamerica), is compared with the Mesoamerican region and that region's favourite institution, the cargo system (that also exists in the Central Andes, although much less studied, under the name of "the fiesta system") under the pressure of neoliberal politics. After a few comments about field work and ethnography, some of the results of the study of the ronda campesina are mentioned, and a second step of that research project is suggested, including a comparison with Mesoamerica and the cargo system.
\end{abstract}

Keywords: the ronda campesina, the cargo system, Mesoamerica, the Central Andes, ethnography of the state

\footnotetext{
* Antropólogo Social de la Universidad de Copenhague, Dinamarca. Doctor en Ciencias Naturales por la Universidad Autónoma Metropolitana en la Ciudad de México. Profesor de Tiempo Completo del Posgrado en Antropología Social de la Escuela Nacional de Antropología e Historia (ENAH), Distrito Federal, México. Correo-e: leifkorsbaek1941@gmail.com
} 


\section{Introducción}

El propósito de este trabajo es discutir algunos problemas relacionados con el estudio antropológico de la institución conocida en el Perú como "la ronda campesina", particularmente con la observación y la descripción etnográfica de esta institución en el contexto de la comunidad indígena y/o campesina, problemas que inevitablemente nos llevan hacia la discusión de otros campos de la antropología.

El problema de la ronda campesina se inscribe en la problemática del pluralismo legal, que ha sido ampliamente tratado por la abogada Raquel Yrigoyen (2003). Sin embargo, la visión aquí presentada es una visión antropológica, y es relevante mencionar que la antropología es una disciplina de la cual existe una abundancia de definiciones, muchas de ellas incompatibles. Sin embargo, los siguientes rasgos parecen ser una especie de denominador común de las variadas definiciones de la antropología: 1) es una disciplina cuyo concepto fundamental es el de "cultura", 2) es la única disciplina dedicada explícitamente al estudio de la alteridad, más exactamente al estudio de la articulación entre la tradición y la modernidad, 3) recoge su información por medio del trabajo de campo, conocido también como etnografía, y 4) mantiene su ambición "holística" (Korsbaek, 2009a:4).

Como planteamos en la convocatoria al simposio donde se presentó originalmente este texto, "es nuestra opinión que un método indispensable para llegar a tener una idea de las características del estado y su dinámica que se acerque a la realidad es el método etnográfico", y leemos en una discusión reciente de las virtudes de la antropología política, que "la antropología es una vía sólida para el estudio del poder político. La evidente fortaleza del cuerpo de conocimiento labrado por la disciplina desde el siglo XIX no deja duda" (Barquín, 2015: 9).

Una de las justificaciones de tal decisión la encontré hace algún tiempo, cuando Polonia era infelizmente estalinista y Zygmunt Bauman era felizmente marxista -y todavía no un sociólogo posmodernista de la Universidad de Columbia en Fifth Avenue, Nueva York- quien escribió:

\begin{abstract}
“(...) si hay algo idiosincrático y peculiar en el enfoque marxista del estudio del hombre, es un esfuerzo obstinado por combinar en un enfoque unificado las múltiples y divergentes imágenes del hombre cuando se le contempla desde diferentes puntos de vista. Utilizando la moderna terminología técnica, cabe decir que la ciencia social marxista procura un holograma del hombre en lugar de una serie de fotografías del mismo. La premisa metodológica básica y, a la vez, el rasgo característico del enfoque marxista de la ciencia social consiste en que el hombre económico, el hombre social, el hombre cultural, el hombre político y otros productos similares de la división científica del trabajo no son sino modelos conceptuales, creaciones de un largo proceso de abstracción madurado en medios microsociales institucionalmente separados. La única realidad auténtica, de la cual parten estos modelos y a la cual se refieren, es el hombre como tal, dedicado al proceso de vivir en su ambiente social y cultural y por medio de este" (Bauman, 1972:17).
\end{abstract}

Sin embargo, la recomendación más incondicional que he encontrado a través de mis años como antropólogo dice que "la antropología es, en muchos aspectos, la más central de las ciencias sociales y ha sido, hasta el momento, una de las más objetivas y la menos políticamente contaminada", en lo que realmente estoy de acuerdo, en efecto, yo mismo podría haber escrito estas palabras, si no fuera por el peligro de parecer ridículo. Nuestra confianza en esta evaluación proviene de una fuente que no puede ser acusada de manejar prejuicios 
a favor de la antropología: las palabras son de un informe confidencial enviado al jefe de el FBI J. Edgar Hoover, acerca del congreso de la Asociación de Antropólogos Americanos (AAA, el gremio profesional de los antropólogos norteamericanos) en Toronto, en 1949, en los mejores tiempos de la guerra fría (Price, 2004).

Por otro lado:

\begin{abstract}
"Hay una ley natural en la antropología: los antropólogos que se dedican a la antropología política raras veces estudian antropológicamente la economía y viceversa, los especialistas en la antropología económica solamente en escasas ocasiones gastan su tiempo en el estudio de la política. Esta ley ya la vemos reflejada en la obra de los dos padres fundadores de la antropología social británica, Bronislaw Malinowski y A. R. Radcliffe-Brown, el primero, un brillante antropólogo económico y el segundo un notable antropólogo político. En concordancia con esta ley, la Escuela de Manchester, que en gran medida debe su fama a sus incursiones en la antropología política, solamente cuenta con una muy tenue presencia en el terreno de la antropología económica, un hecho que ya se vislumbra en la biografía del fundador de la Escuela de Manchester, el antropólogo Max Gluckman" (Korsbaek, 2016: 75).
\end{abstract}

Quisiera mencionar que el texto citado arriba tiene que ver al mismo tiempo con el pasado de mis actividades como investigador y con el futuro de las mismas actividades. Por lo menos en dos puntos tiene que ver con mi pasado antropológico: durante muchos años me he dedicado a estudiar la institución conocida como el sistema de cargos, que "ha recibido la atención de prácticamente todos los antropólogos que han trabajado en la región" (Cancian, 1967:283), y a partir de 2007, cuando pasé mi año sabático como profesor invitado de la Universidad de San Marcos en Lima, en el Perú, me he dedicado a estudiar otra institución conocida como la ronda campesina.
Es claro que ambas instituciones pertenecen a "las instituciones de defensa de la comunidad", tal como las he estudiado en otro contexto (Korsbaek, 2009b).

La ronda campesina es claramente una institución jurídica, y en otro contexto he señalado que es también una institución política. La razón por la cual inicié el presente texto con la situación de la economía y la política es que difícilmente la podemos acusar de ser una institución económica. Es cierto que todo tiene un aspecto económico, así como también la ronda campesina; Edmund Leach menciona -con su encantador cinismo que le granjeó varios enemigos en la antropología social británica- que el sacramento tiene su aspecto económico, pues alguien tiene que proporcionar el pan y el vino, mientras que Malinowski menciona en lo que habría sido su primera conferencia pública, que "la ceremonia Intichiuma tiene su aspecto económico" (Malinowski, 2015).

El presente texto tiene algo que ver con el Estado, tal vez de una manera negativa. En otra ocasión he escrito que "los antropólogos somos los únicos que saben de la existencia de un proceso político sin la existencia del estado", y en mi introducción del concepto de "instituciones de defensa de la comunidad" he escrito que forzosamente tenemos que tomar en cuenta la existencia del Estado y su influencia (Korsbaek, 2009b: 373).

En síntesis, en el presente texto quisiera tratar dos propuestas, ambas en la forma de una hipótesis, aunque en diferentes niveles. En primer lugar, quisiera recomendar una etnografía de la ronda campesina cuyos fundamentos tienen su origen en la tradición británica de la antropología, un punto que elaboraré más adelante 
en el texto. En segundo lugar, quisiera plantear un enfoque en el estudio de la ronda campesina que tiene que ver con la relación entre la región de Mesoamérica y la región andina, un punto que elaboraré también con más detalles adelante.

\section{La ronda campesina}

La ronda campesina es, antes que nada, una institución que pertenece a la comunidad campesina o indígena: "son órganos de vigilancia y protección propia de las comunidades campesinas; su función es cautelar la vida, la integridad y el patrimonio de los comuneros frente a la existencia de posibles actos que vulneren sus derechos fundamentales" (Machaca, 2000:9). Según otros autores,

"(...) son organizaciones de campesinos que en forma voluntaria realizan labores comunales de seguridad de sus pueblos, resuelven pacíficamente los conflictos en sus comunidades y participan activamente en el desarrollo de sus localidades" (Laos, Paredes \& Rodríguez, 2003:13).

\section{Mientras que para la Defensoría del Pueblo}

"(...) las rondas campesinas son formas de organización comunal y campesina, que representan y organizan la vida comunal, ejercen funciones de justicia, interlocución con el estado y realizan tareas de desarrollo, seguridad y paz comunal, dentro de su ámbito territorial" (Defensoría del Pueblo, 2004:13).

Es sabido que las primeras rondas surgieron en 1976 en Chota, Cajamarca, en el norte del Perú, y se difundieron como un incendio en la pradera, hasta llegar a números increíbles, arriba de 100.000 ronderos, tan solo en la provincia de Cajamarca. Más precisamente, "la primera ronda se fundó en Cuyumalca, estancia contigua a la ciudad de Chota, a las 2 p.m. del
29 de diciembre de 1976. Esta histórica decisión la tomaron los padres de familia de la escuela, y fue ratificada en días siguientes por todas las familias de Cuyumalca. El nombre original fue Rondas Nocturnas" (Rojas, 1990:89), como se desprende del Acta Histórica de la Fundación que reza como sigue:

"En la estancia de Cuyumalca, siendo las 2:00 pm. del día veintinueve de diciembre de mil novecientos setenta seis, reunidos los ciudadanos de dicha comunidad, luego de intercambio de ideas se llegó al acuerdo de organizar "Rondas Nocturnas" para defender los intereses del centro educativo y de toda la comunidad a consecuencia de los continuos robos que se vienen suscitando en agravio de dicho centro y de algunos vecinos. Esta acta tiene la finalidad de organizar a la comunidad y solicitar la licencia respectiva a fin de que sea posible comprar sus armas. El encargado de organizar las rondas será el Teniente Gobernador, quien previo empadronamiento de la ciudadanía, distribuirá el personal; será también el encargado de seguir los trámites correspondientes para el buen desempeño de sus funciones. Con lo que se dio por terminado el acta siendo las 3:15 pm. Copia de la presente acta será remitida a las autoridades respectivas de la provincia a fin de solicitar garantías y parejas de Guardias Civiles cuando el caso lo requiera. Asimismo, la comunidad acordó dirigirse, mediante un memorial, al Presidente de la Corte Superior de Justicia de Lambayeque pidiendo el nombramiento de un juez único de primera nominación por intermedio del juez instructor de nuestra provincia".

Lo anterior corresponde al momento de nacimiento de la ronda campesina, su origen histórico. Pero quedándonos en la dimensión histórica podemos descubrir algunos antecedentes de esta institución: según algunos, "en las rondas parecen confluir tres vertientes institucionales: las guardias de las haciendas por su función, la comunidad campesina, por su organización, y el servicio militar obligatorio, como requisito para ejercer algunos roles", según otros: 
“(...) es posible afirmar que no existe sino un solo fenómeno - a diferencia de lo que creen algunos estudiosos de las rondas - que se puede considerar como antecedente de esta organización: las guardias campesinas de las haciendas, encargadas por el hacendado de vigilar su propiedad y de perseguir a los abigeos" (Zarzar, 1991:108).

Luego, una "serie de factores confluyen en el surgimiento de las rondas campesinas. La crisis económica, el abigeato, la corrupción de la justicia y el vacío de autoridad estatal aparecen mencionados de modo prácticamente unánime en los estudios" (Degregori \& Ponce, 2000: 395). Mientras que Orin Starn añade otra razón: "la recompensa cultural que en el campo norteño tiene el ser rudo, terco y temerario" (Starn, 1991:38).

Es evidente que en tal cantidad de instituciones que existen en regiones muy alejadas, de muy diferente carácter y con trayectorias históricas muy diversas, no todas las rondas pueden ser idénticas, deben de existir diferentes tipos, lo que sí es evidente es la distinción de cuatro tipos de rondas campesinas.

Un primer tipo de rondas campesinas son aquellas surgidas sobre la base de los caseríos, donde no han existido comunidades campesinas, como en el caso de Cajamarca, San Martín y Amazonas, fundamentalmente. Un segundo tipo son las que han surgido dentro de las comunidades campesinas, como es el caso en Piura, Ancash, La Libertad y en el sur andino (casi todas las rondas campesinas de Carabaya en Puno y de las provincias de Espinar y Canas y del distrito de Quispicanchis en Cusco), que cumplen el papel de órgano auxiliar de las comunidades y dependen de estas. Un tercer tipo son aquellas rondas campesinas que existen en comunidades nativas de la selva peruana.
$Y$, finalmente, un cuarto tipo son los denominados "comités de autodefensa" que nacieron en la década de los noventa impulsados por el Estado para la lucha contra la subversión en la época de la violencia política, y que han surgido indistintamente al interior de las comunidades campesinas y parcelas o caseríos donde no hay presencia de comunidades campesinas (Rodríguez Aguilar, 2007).

El primer tipo de rondas campesinas, aquellas que han surgido sobre la base de los caseríos

“(...) surgen en una región abandonada por el Estado. Esta ausencia, sin embargo, no es ninguna peculiaridad de la sierra norte. Lo peculiar en la sierra norteña es más bien que los campesinos no contaron con mecanismos sociales propios para contrarrestar este vacío de autoridad y sus consecuencias. La ausencia de un organismo regulador se manifestó sobre todo en la vida cotidiana; la región era afamada por peleas sangrientas, hurtos de ganado, que aumentaron después del retiro de los hacendados" (Huber, 1995:121-122).

No me parece que se trate de "una región abandonada por el Estado", pues, por un lado, se trata de un proceso histórico que ha producido una situación en la cual "el departamento de Cajamarca, como toda la sierra norteña del Perú, es tierra de campesinos parcelarios", y "en Cajamarca el número de comuneros, cuyas tierras oficialmente son propiedad de la comunidad en su conjunto, apenas llega al $0.6 \%$ de la población campesina" (Huber \& Guerrero, 2006:13). Por otro lado, un fuerte impulso que contribuyó a la producción de esta situación, lo tenemos que buscar en la reforma agraria de Velasco Alvarado, tal como señala Carlos Iván Degregori:

"(...) las rondas de Cajamarca y Piura surgieron teniendo como telón de fondo la desaparición de los terratenientes luego de la reforma agraria, y la multiplicación de los parcelarios libres. En Piura, la propiedad 
individual se encontraba revestida bajo la etiqueta de comunidad campesina" (Pérez, 1994; Huber, 1995).

Pero esta era más una formalidad, sin los lazos de ayuda mutua, trabajo colectivo y asambleas que suelen caracterizar como tipo ideal a las comunidades de los Andes centrales. En este contexto, la organización rondera logró desarrollar una fuerte identidad colectiva en la que muchos encontraron similitudes con las comunidades campesinas de otras partes de la sierra (Degregori \& Ponce, 2000). Resumiendo, en una parte de Cajamarca, exactamente donde surgió en 1976 la primera ronda campesina, tenemos una situación en la cual no existe la tenencia comunal de la tierra, donde los campesinos son campesinos parcelarios y no existe una larga tradición de una toma de decisiones colectivas; en este ambiente la ronda campesina llega a llenar un vacío político y constituirá algo así como una estructura social de la comunidad campesina.

El segundo tipo surge en regiones donde las condiciones son radicalmente diferentes, donde los campesinos viven en comunidades con una sólida tradición colectiva que se enmarca en un contexto de tenencia comunal de la tierra:

"El III Censo Nacional Agropecuario de 1994 revela que en el departamento de Apurímac existían 438 comunidades campesinas registradas que poseían 1.228.711 hectáreas de tierra y la mayor parte de dicha población rural vivía en estas unidades de propiedad comunal de la tierra (81\% en Apurímac)" (Ramírez Salazar, 2007:25).

\section{De manera que:}

"Las rondas campesinas del sur andino, en tanto organizaciones distintas a las que existen en el resto del país, así como, por haberse desarrollado en un contexto político, social, histórico e incluso cultural, diferente a las rondas del norte y centro, podrían definirse como órganos de vigilancia, protección y justicia de las comunidades campesinas, ayllus, centros poblacionales y parcialidades, para ejercer funciones relacionadas a la seguridad comunal, justicia comunal restaurativa, promover el desarrollo comunal, y establecer relaciones de coordinación y diálogo con las instancias del Estado, dentro del ámbito territorial donde se organizan, conforme a los valores, principios y creencias de su propia lógica y racionalidad' (Rodríguez Aguilar, 2007:16).

El tercer tipo, "las rondas campesinas de comunidades nativas de la selva peruana" (Rodríguez Aguilar, 2007: 17-18), en muchos casos han tenido que coexistir con el narcotráfico y otras actividades ilícitas que existen debido a la casi total ausencia del Estado y de sus representantes. Una descripción romántica de la ausencia del Estado en la selva amazónica es la famosa novela Pantaleón y las visitadoras de Mario Vargas Llosa, un excelente novelista y un miserable pensador político; una impresión mucho menos romántica de la ausencia del Estado y de cualquier normatividad en la selva amazónica se puede tener de la situación en las orillas de la carretera de Cusco a Puerto Maldonado, con la explotación despiadada en las minas de oro y la prostitución infantil en los campamentos ${ }^{1}$.

El cuarto tipo, los denominados "comités de autodefensa", es probablemente el más problemático, como se señala en el informe final de la Comisión de Verdad: "En ningún otro actor de la guerra, la línea divisora entre perpetrador y víctima entre héroe y villano, es tan delgada y porosa como en los comités de autodefensa (CAD) o rondas campesinas contrasubversivas"(Lerner, 2003:13).

La obra de Carlos Iván Degregori y otros (1990), acerca de la contribución de las rondas 
campesinas a la derrota del movimiento de rebelión "Sendero Luminoso" es un importante aporte al estudio de la dialéctica entre los diversos tipos de la ronda campesina, pues trata cuatro instancias de la contribución, tanto de las rondas campesinas que podemos llamar auténticas, como de los comités de autodefensa, en aplacar la violencia en el ambiente de comunidades campesinas en el Perú.
En este sentido, respecto a los veinte años de violencia en el Perú, entre 1980 y 2000 :

"Para el año 1993, el éxito de los Comités de Autodefensa era más que notable por su satisfactoria respuesta a las necesidades de seguridad de las comunidades, y también por su protagonismo en la organización política de las localidades. El siguiente cuadro muestra la distribución e importancia numérica de estos grupos" (Castañeda, s. f.:1-2).

\begin{tabular}{|l|l|r|r|r|}
\hline $\mathbf{N}^{\circ}$ & DEPARTAMENTO & CAD & INTEGRANTES & ARMAS \\
\hline $\mathbf{1}$ & Tumbes & 70 & 2871 & \\
\hline $\mathbf{2}$ & Piura & 282 & 17778 & 194 \\
\hline $\mathbf{3}$ & Lambayeque & 105 & 5730 & 916 \\
\hline $\mathbf{4}$ & Cajamarca & 443 & 27927 & 858 \\
\hline $\mathbf{5}$ & La Libertad & 219 & 14018 & 142 \\
\hline $\mathbf{6}$ & Ancash & 27 & 1350 & 3855 \\
\hline $\mathbf{7}$ & Junín & 525 & 34537 & 626 \\
\hline $\mathbf{8}$ & Cerro de Paso & 68 & 6072 & 5583 \\
\hline $\mathbf{9}$ & Ayacucho & 1564 & 61450 & 646 \\
\hline $\mathbf{1 0}$ & Huancavelica & 198 & 10658 & 508 \\
\hline $\mathbf{1 1}$ & Lima & 33 & 2869 & 160 \\
\hline $\mathbf{1 2}$ & Puno & 86 & 3627 & 260 \\
\hline $\mathbf{1 3}$ & Loreto & 30 & 911 & 100 \\
\hline $\mathbf{1 4}$ & Amazonas & 112 & 8295 & 440 \\
\hline $\mathbf{1 5}$ & Apurímac & 63 & 3618 & 60 \\
\hline $\mathbf{1 6}$ & Cusco & 106 & 5802 & 868 \\
\hline $\mathbf{1 7}$ & Madre de Dos & 20 & 1232 & 892 \\
\hline $\mathbf{1 8}$ & Huanuco & 163 & 13578 & 170 \\
\hline $\mathbf{1 9}$ & San Martín & 55 & 11658 & $\mathbf{1 6 1 9 6}$ \\
\hline $\mathbf{2 0}$ & Ucayali & 36 & $\mathbf{2 3 5 4 6 5}$ & \\
\hline $\mathbf{T O T A}$ & $\mathbf{4 2 0 5}$ & & & \\
\hline
\end{tabular}




\section{Un postulado acerca del Estado}

El Estado moderno es implacable, existen solamente dos tabicones primordiales: el Estado y el ciudadano. El Estado moderno y la sociedad moderna se distinguen de la sociedad tradicional, y su Estado, por un número de rasgos: el Estado (y la sociedad) moderno es materialista, secular e individualista, mientras que la sociedad tradicional es mucho menos individualista y más colectivista, es religiosa y espiritual.

El mundo moderno, que hoy estamos disfrutando/padeciendo, es producto de la revolución moderna, una triple revolución que se llevó a cabo entre 1350, el año del fallecimiento de Guillermo de Ockham, y 1650, el año del fallecimiento de Descartes: el Renacimiento, que dotó al ser humano de una nueva sensibilidad, el nacimiento del capitalismo, que le dotó de una nueva racionalidad, no solamente en lo económico, sino también en lo político, en lo social y en lo ideológico, y el descubrimiento mutuo del Nuevo Mundo y el viejo, creando así por primera vez en la historia de la humanidad un mundo redondo.

Para iniciar una discusión de lo que llamo "el mundo moderno", con el Estado moderno, me permito citar un largo trozo de mi tesis doctoral (Korsbaek, 2009a: 9-12):

\footnotetext{
"Quisiera destacar dos importantes fuentes de inspiración de esta investigación. En primer lugar, la obra de Max Gluckman y la Escuela de Manchester. La contribución más importante de Max Gluckman es haber introducido al conflicto como objeto de estudio en la antropología. Su declaración más importante es: "Creo que el conflicto y la superación del conflicto (fisión y fusión) son dos aspectos del mismo proceso social que están presentes en todas las relaciones sociales (Gluckman, 1958: 26)".
}

Otra contribución de Gluckman que salta a la vista es su introducción del estudio antropológico de la revolución, estrechamente relacionada con la distinción entre la "revolución" y la "rebelión", lo que nos lleva a otra característica de su antropología: su relación con los estudios de Evans-Pritchard, a quien se debe originalmente dicha distinción. En varios casos, Max Gluckman ha retomado conceptos inicialmente introducidos por Evans-Pritchard, pero dándoles un significado y un uso fundamentalmente distintos. El conflicto -que curiosamente ha estado permanentemente presente en la antropología, ya que los antropólogos normalmente trabajan en regiones muy conflictivas - entró tardíamente como objeto de estudio en la antropología, muchos años después de que ya se había presentado en la sociología como parte de su herencia original (Coser, 1961).

La segunda influencia decisiva es la obra de Louis Dumont. En otro lugar he formulado mi concepción del mundo moderno y del tradicional, pero sin poder hacer referencia a las investigaciones de Louis Dumont, probablemente no me habría atrevido a fundamentar la lógica de la investigación en el postulado de la coexistencia de un mundo tradicional - en la terminología de Dumont, la "jerarquía"- y el mundo moderno que Dumont llama "individualismo".

Este debe ser el lugar para presentar las hipótesis que guían la investigación, pero antes quisiera citar a Max Gluckman una vez más:

\footnotetext{
"Yo también soy evolucionista en el sentido de que considero que cuando evaluamos e intentamos entender el significado de las instituciones es esencial examinarlas contra el trasfondo de lo que sin lugar a dudas ha sido una de las más grandes tendencias en la historia de la sociedad humana en su totalidad -la creciente complejidad de la tecnología y, con ella, la creciente complejidad de la organización económica.
} 
Yo creo que cuando nos representamos las formas de la organización social y de las creencias e ideas sociales asociadas a diferentes rangos de tecnología, iluminamos a todas. Esa es ahora una línea de pensamiento poco popular entre los antropólogos sociales, y así ha sido durante mucho tiempo, la única excepción siendo la escuela bajo el Profesor Leslie White en la Universidad de Michigan. Durante años recientes el énfasis se ha colocado en lo que es común en todas las instituciones de las sociedades humanas, y es cierto que hay muchas cosas que todas las sociedades comparten. Sin embargo, dentro de esta área común demarcada es posible registrar diferencias que adquieren importancia de su mátrix compartida en la vida social" (Gluckman, 1972:1).

La hipótesis más general postula que en el mundo existen dos diferentes maneras de pensar y actuar, como señalaba respecto a Louis Dumont, una que podemos llamar "jerarquía" y otra que podemos llamar "individualista", que el autor define de la siguiente manera: en la sociedad tradicional rige un ambiente colectivista, el individuo tiene existencia principalmente en cuanto miembro de una colectividad, la "comunidad", mientras que en la sociedad moderna, "el ser humano es considerado como el hombre indivisible, elemental, como un ser biológico y al mismo tiempo como un sujeto pensante". Los textos más tempranos que conozco de Dumont son de alrededor de 1970, pero es fácil encontrar sus antecesores, podemos decir que es una modernización del principal planteamiento de Henry S. Maine, donde distingue entre sociedades tradicionales con estatus adscritos y sociedades modernas con estatus adquiridos. Podemos sospechar un desarrollo sostenido desde el mundo tradicional hacia el mundo moderno, aunque sí tenemos que aceptar la posibilidad de reversiones de este movimiento. Sin embargo, mientras que los evolucionistas suponen que se dé un movimiento a través del tiempo, y además unidireccional y unilineal, supongo la coexis- tencia de elementos de los dos mundos en la sociedad en un solo momento dado.

La segunda hipótesis, postula que el sistema de cargos es la institución dominante en la comunidad, que sea indígena o campesina, tampoco una hipótesis muy original. Pero sí, con cierta justificación podemos suponer que el sistema de cargos sea la institución que le asigna a los elementos su valor culturalmente específico y que en cierta medida determine el rumbo del desarrollo de la comunidad. Si es cierto que la comunidad se encuentra como eslabón entre el mundo moderno y el tradicional, y que el sistema de cargos sea la institución central en la comunidad, entonces podemos también esperar que el sistema de cargos esté sometido a cierta presión que lo empuje hacia la modernización. Podemos buscar en el sistema de cargos una dinámica que no es necesariamente una resistencia contra el proceso de modernización, se manifiesta como una institución que dota a la comunidad de cierta autonomía en cuanto a la dirección a asumir bajo la presión de las fuerzas centrífugas.

La tercera hipótesis plantea que en el marco del sistema de cargos, el proceso de modernización asume la forma de individualización. Podemos formular la situación de la siguiente manera: en el mundo tradicional, el individuo está sometido y guiado por el sistema de cargos, mientras que en el mundo moderno, el sistema de cargos está sometido a los deseos y las ambiciones del individuo.

En vista de la enorme cantidad de estudios que existen del sistema de cargos se podría pedir una justificación del presente estudio, otro estudio de una institución ya tan estudiada; espero que no sea como opina Darcy Ribeiro, 
que "en verdad, los científicos sociales están preparados para hacer estudios cuidadosos y precisos sobre temas restringidos y en último término irrelevantes" (Ribeiro, 1977:9-10). Para justificar una investigación como la que aquí se plantea se pueden invocar los siguientes "puntos", siendo exactamente los que tienen que ver con la separación (y articulación) del mundo moderno y el tradicional.

\section{Dos instituciones, dos regiones}

Mencioné anteriormente que he trabajado en dos regiones separadas por una muy larga distancia y que, a pesar de sus grandes diferencias, comparten varios rasgos: Mesoamérica y particularmente en la parte central de la región andina.

En 1996 fue publicada la antología Introducción al sistema de cargos, en la cual fueron presentados un hermoso prólogo de Andrés Medina Hernández, mi traducción (del inglés al español) de ocho textos acerca de la institución conocida como el "sistema de cargos" -que podemos tildar de clásicos u ortodoxosademás de una bibliografía en aquel entonces razonablemente completa y dos capítulos interpretativos, en el primero de los cuales se presentó la idea de un "típico sistema de cargos" y en el segundo la idea del "paradigma del sistema de cargos".

Los objetivos de la antología fueron formulados del siguiente modo: "presentar sistemáticamente a la institución social y cultural que ha sido nombrada de diferentes maneras: "el sistema de fiestas" (Smith, 1981) "el sistema de cargos" (Tschopik, 1947), "el sistema de vara" (Carrasco, 1979), "el sistema de escalafón"
(Cancian, 1967), "la jerarquía político-religiosa" Cámara Barbachano, 1952) y otros más.

A partir de la primera descripción antropológica de esta institución, realizada en $1937^{2}$, se ha venido cambiando radicalmente la situación: desde su virtual inexistencia antropológica, antes de ese año, hasta llegar a ser considerada como "la institución político-religiosa característica en las comunidades indígenas de Mesoamérica" (Cancian, 1967; Cámara Barbachano 1952), comparable con los fenómenos del sistema de castas en la India, el potlatch entre los indígenas de la Costa Pacífica de Canadá, los sistemas de parentesco unilineal en las sociedades africanas, etcétera. El descubrimiento de esta institución vino a cambiar por completo el ambiente cultural y antropológico en Mesoamérica, y nadie expresa mejor que Manning Nash el resultante entusiasmo y la opinión general de los antropólogos, en lo que a la importancia del sistema de cargos se refiere: "la jerarquía es, prácticamente, toda la estructura social del municipio. Al nivel más general de integración social, esta institución representa para los indios lo mismo que el parentesco para las sociedades africanas y el sistema de clases sociales para las sociedades ladinas" (Nash 1958: 165).

El típico sistema de cargos fue definido así:

"El sistema de cargos consiste en un número de oficios que están claramente definidos como tales, que se turnan entre los miembros de la comunidad quienes asumen un oficio por un período corto de tiempo después de lo cual se retiran a su vida normal por un período de tiempo más largo. Los cargueros no reciben pago alguno durante su período de servicio, por el contrario, muy a menudo el cargo significa un costo considerable en tiempo de trabajo perdido y en gastos en dinero en efectivo, pero como compensación el cargo confiere al responsable un gran presti- 
gio en la comunidad. Los oficios están ordenados jerárquicamente y el sistema de cargos comprende a todos - o casi todos - los miembros de la comunidad. El sistema de cargos comprende dos jerarquías separadas, una política y una religiosa, pero las dos jerarquías están íntimamente relacionadas, y después de haber asumido los cargos más importantes del sistema un miembro de la comunidad es considerado como "pasado" o "principal" (Korsbaek, 1996: 182)³.

El "paradigma del sistema de cargos" se presentó de la siguiente manera:

"El paradigma de cargos es, en lo que se refiere al aspecto económico, claro y sencillo y se desprende con facilidad de la etiqueta que los antropólogos le han otorgado al sistema de cargos en su calidad de institución económica: se conoce como "mecanismo nivelador" ("levelling mechanism"4).

Como regla general se supone que el sistema de cargos cumple la función de redistribuir la riqueza producida en la comunidad entre sus miembros y de esta manera obstaculizar la acumulación de capital y la monopolización de la riqueza. La gran mayoría de los antropólogos que han estudiado el sistema de cargos expresan esta opinión, con muy diferentes grados de generalidad y de claridad y a diferentes niveles de abstracción:

"En el aspecto político del paradigma se postula que el sistema de cargos es una institución netamente democrática. El aspecto religioso del paradigma es, finalmente, más complejo y lo podemos dividir en cuatro postulados: 1) define a la comunidad, es decir produce la identidad étnica y comunitaria, 2) define las fronteras de la comunidad, 3) define los canales legítimos de comunicación entre la comunidad y las autoridades eclesiásticas y políticas del sistema nacional, y 4) finalmente es una institución conservadora que se opone a cualquier cambio en la comunidad" (Korsbaek,1996: 271-292).
Todo eso estuvo muy bien hasta alrededor del año 1968, tal vez un poco antes, cuando empezó una nueva etapa del estudio antropológico del sistema de cargos (y de muchas otras cosas, dentro y fuera de la antropología).

Hasta ahora me he referido principalmente a la antropología en México, pero la antropología en Guatemala ocupa también un lugar notable en el estudio del sistema de cargos, y por lo menos tres factores merecen mencionarse. En primer lugar, existe una serie de estudios tempranos que tienden a considerar al sistema de cargos (que todavía no había sido introducido en la antropología y menos había sido bautizado) como una institución casi exclusivamente religiosa, con fuertes raíces en el mundo de la preconquista (un ejemplo de este interés por la preconquista es Lafarge \& Byers, 1931), una tendencia que siguió manifestándose más tarde también. En segundo lugar, al final de la Segunda Guerra Mundial, en los tempranos años de la Guerra Fría, Guatemala fue invadida por antropólogos norteamericanos con una visión cristera de la antropología como un arma en la lucha contra el comunismo ${ }^{5}$. Esta lucha anticomunista llega a su clímax con la caída de Jacobo Arbenz en 1954, y la obra clave es Political Change in Guatemalan Indian Communities. A Symposium, coordinada por Richard Newbold Adams y con participación de la crema y nata de antropólogos norteamericanos en Guatemala (Adams, 1957) ${ }^{6}$. En tercer lugar, en esta antropología hay una fuerte representación de la antropología psicológica, una especialidad que es relativamente débil en el estudio antropológico del sistema de cargos en México. Se destacan aquí las monografías de John P. Gillin, Melvin Tumin, un artículo de Benjamín Paul (1976) y, un poco más tarde, de Mendelson?. 
Sin embargo, con el transcurso de los años los trabajos de campo han tendido a subrayar y confirmar la distancia que existe entre los planteamientos ideales, supuestamente objetivos, del paradigma del sistema de cargos y una realidad empírica rebelde, de los tres principales niveles de análisis: económico, político y religioso. En lo económico, Frank Cancian mostró, con las cifras de Sol Tax, que no es posible la nivelación que postuló Tax en Panajachel (Cancian, 1989); lo que mostró Robert Wasserstrom en el caso de Zinacantán (1977) en lo político, mientras que, Henning Siverts (1964, 1965b, 1969b) y Evon Z. Vogt (1963) han mostrado el lugar que ocupa el cacique en la política del sistema de cargos; en lo religioso, nadie ha comprobado más contundentemente que Paul Diener (1978) que el sistema de cargos no solamente no se opone al cambio, sino que era positivamente, en algunos casos, el fundamento de las actividades guerrilleras.

Muy tempranamente, se sembraron dudas acerca de la realidad del supuesto proceso de nivelación. En la década de 1960, Frank Cancian desarrolló una crítica a la interpretación niveladora de los antropólogos (Cancian, 1967, 1989), proponiendo el modelo de estratificación y desde entonces creo que nadie toma en serio la supuesta función niveladora del sistema de cargos. Según Cancian, lo que hace el sistema de cargos no es evitar las desigualdades económicas, pues estas serían importantes para la integración de la comunidad: lo que hace es legitimarlas ${ }^{8}$. Este modelo, que es mucho más sofisticado y menos romántico que el anterior modelo de nivelación, ha sido adoptado por varios antropólogos críticos, como Juan Castaingts (1979), Waldemar Smith (1981), Chance y Taylor (1985) y James Green- berg (1987). Junto con la aplicación del modelo de estratificación se desarrolla la dimensión histórica (sobre todo en los trabajos de Chance y Taylor, y de Juan Castaingts), y una protesta contra el carácter cerrado de los estudios de comunidad (sobre todo en el trabajo de Waldemar Smith).

También se cuestionan algunos de los elementos religiosos en el paradigma de cargos. Por ejemplo, acerca de su supuesto carácter conservador y su resistencia al cambio social, Paul Diener publicó en 1978 un artículo en el cual documentó que el sistema de cargos en algunos casos en Guatemala no solamente no se oponía a los cambios "propuestos" por la guerrilla en aquel país, sino que constituía el esquema organizacional de la misma guerrilla. El núcleo del aspecto político del paradigma de cargos fue el postulado de su carácter inherentemente democrático, tal como fue planteado con su acostumbrada elegancia por Eric Wolf.

Antes de continuar, quisiera mencionar un importante texto de Pedro Carrasco, de 1961, en el cual advierte respecto a confundir la dinámica de la estructura social de una institución, su forma cultural y su función, pues los tres aspectos tienen cada uno su relativa autonomía e independencia. Vale la pena mencionar que, en el mismo texto, Carrasco le otorgó al sistema de cargos una dimensión histórica que la cargología de inspiración británica le había negado, sugiriendo que el sistema de cargos era una institución de materia prima cultural de la preconquista que había sido refomulada, refuncionalizada y reconstruida al calor de la conquista y en la temprana colonia, por la conquista espiritual.

El primer misterio en este contexto refiere a que si el sistema de cargos es tan democrático 
como dicen, entonces ¿cómo es posible que coexista tan armoniosamente con cualquier tipo de caciquismo? Los ejemplos abundan, pero a título de ejemplo podemos mencionar el caciquismo en San Juan Chamula (Vogt, 1973), aún antes de los eventos zapatistas, durante la paz priista en Chiapas, y entre los triques en Oaxaca (Huerta, 1997).

Es evidente que la crítica al aspecto político del paradigma de cargos seguía desarrollándose y ampliándose, pero salta a la vista que la investigación del aspecto político del sistema de cargos es la parte más débil de la cargología.

Es cierto que Henning Siverts ya en los años sesenta señaló que "este sistema de cargos que en realidad constituye el gobierno semiautónomo en un buen número de pueblos indígenas en Chiapas y en otras partes" (Siverts, 1965a: 387), pero poco tiempo después declaró que "todavía no tenemos un estudio detallado del carácter político del sistema de cargos" (1965a: 340). En este texto mi intención es tan solo comentar acerca de algunos aspectos de la cargología política, haciendo referencia a una serie de textos relativamente poco conocidos al respecto, pisando así un terreno que es al mismo tiempo bien trabajado y relativamente desconocido.

Aproximadamente a partir de 1968, un año en muchos sentidos emblemático, nos encontramos en una situación algo incómoda en lo que se refiere al sistema de cargos en la antropología: el sistema de cargos no existe solamente en Mesoamérica, no se encuentra solamente en comunidades campesinas y no solamente lo encontramos entre indígenas, aparentemente no nivela nada, no ahuyenta a los caciques y de ninguna manera se opone al cambio social y cultural, ocasionalmente todo lo contrario, y tampoco podemos estar completamente segu- ros de que el clásico sistema de cargos sea típico. Sin exagerar, podemos declarar la existencia de un escándalo científico (para utilizar este concepto de Thomas Kuhn, 2007, originalmente de 1971).

Quisiera mencionar un texto ausente. El primer texto en el cual fue presentado el típico sistema de cargos, de Noriega Hope, es realmente una reliquia, pues data de 1922, un año emblemático en la historia mundial de la antropología, sobre todo de la británica, año en que sucedieron cuatro eventos de primera importancia. En primer lugar, la muerte de W. H. R. Rivers, la figura más importante, y además carismática, en la antropología británica durante los años anteriores, en segundo lugar, fueron publicadas las dos monografías isleñas de los dos padres fundadores de dicha antropología británica, The Andaman Islanders de Radcliffe-Brown y The Argonauts of the Western Pacific de Bronislaw Malinowski, y finalmente, como una contribución de primera a la etnografía mundial, fue publicada la novela Ulises de James Joyce, un tour de force etnográfico basada en veinticuatro horas en la vida de Stephen Daedalus en Dublín. En México fue publicado el mismo año 1922, Teotihuacan, de Manuel Gamio, una contribución a la arqueología y al turismo en México, y a un gran número de prejuicios políticos y científicos que en muchos casos perduran hasta hoy. En esta obra es que encontramos el artículo de Carlos Noriega Hope (1922). Mientras que la introducción ortodoxa al sistema de cargos ubica la invención del sistema de cargos en general, y el típico sistema de cargos, en particular, en 1937, en el ya clásico artículo de Sol Tax de Guatemala; aunque realmente podemos buscar las primeras huellas del típico sistema de cargos en el texto de Noriega Hope, ya mencionado. Sin embargo, como ya señalé 
en otra ocasión, ya que el artículo de Noriega Hope no sería conocido más allá de un muy estrecho círculo, podemos seguir considerando el artículo de Sol Tax como la primera presentación del típico sistema de cargos.

Conviviendo durante algunos años, primero con el sistema de cargos, y luego con la ronda campesina, es casi inevitable plantearse la pregunta acerca de si hay alguna relación entre las dos instituciones, aparte del hecho de que ambas son instituciones de defensa de la comunidad, y en efecto, me parece que sería posible relacionar las dos instituciones dentro de un solo proceso histórico. En el contexto del Congreso Internacional de los Americanistas en Viena en 2012 se presenta:

\footnotetext{
“(...) un proceso histórico en el cual una serie de instituciones comunitarias, que podemos llamar tradicionales, se convierten, bajo el impacto del neoliberalismo, en instituciones militantes que se dedican netamente a la defensa de la comunidad, elemento del mundo tradicional indígena y campesina, contra el estado neoliberal que representa los intereses del mundo moderno capitalista" (Korsbaek, 2012:1).
}

Con eso se plantea una segunda etapa del proyecto de investigación que se cerrará con la publicación del libro de la ronda campesina, un proyecto que toma como su punto de partida el hecho de que el Estado se ha convertido en lo que Noam Chomsky ha denominado como un "estado fallido" y que pretende prestar atención a los varios aspectos de las instituciones de defensa de la comunidad, en particular su papel de institución legal y política. Antes de cerrar con unos comentarios finales, quisiera agregar una breve discusión de algunos de los detalles de la etnografía.

\section{Etnografía y trabajo de campo}

En base a lo anterior queda más o menos claro cuáles son las intenciones y pretensiones del proyecto planteado y, antes de llegar a formular unas conclusiones quisiera intercalar unas reflexiones acerca del trabajo de campo que se hace necesario en el proyecto.

Tenemos en nuestras manos dos regiones con dos instituciones diferentes, pero con similitudes y, además, con una relación específica, pues se plantea hipotéticamente que una proviene históricamente de la otra, bajo condiciones históricas dadas, o sea bajo las condiciones que impone el neoliberalismo dominante en ambas regiones.

Con eso llegamos a plantear la necesidad de observar y describir el neoliberalismo en las dos regiones, como un primer paso. Esa es ya una tarea factible, pues tenemos a nuestra disposición un acervo de experiencias en estudios del Estado neoliberal en su contexto social y cultural. Es claro que hay obstáculos.

Pierre Bourdieu menciona un peligro que amenaza cualquier intento por observar, pensar o describir el Estado: "atreverse a pensar en el estado es asumir el peligro de coger (o ser cogido por) el pensamiento del estado, es decir aplicar al estado las categorías de pensamiento producidas y garantizadas por el estado, y así errar en la búsqueda de su verdad más profunda", y prosigue "una de las principales fuerzas del estado es el producir e imponer (sobre todo por medio del sistema de educación) categorías de pensamiento que aplicamos espontáneamente a todas las cosas en el mundo social - incluyendo el estado mismo" (Bourdieu, 1994:1). 
Pero tenemos una complicación más, producida por un sociólogo ya hace algunos años:

"El estado no es la realidad que se esconde detrás de la máscara de la práctica política. El estado es él mismo la máscara que no nos permite ver la práctica política tal como es. Hay en una sociedad dada un sistema estatal, un nexo palpable de práctica y estructura institucional centrada en el gobierno, y más o menos extensivo, unificado y dominante en la sociedad en cuestión. $Y$ hay una idea-estado que es proyectada, proporcionada y en diferentes grados creída en diversas sociedades en diferentes momentos. Solamente nos creamos dificultades si suponemos que tenemos también que estudiar el estado - una cosa, agente, función, relación, más allá del sistema estatal y la idea-estado. El estado llega a existir a través de la práctica política, su vida empieza como un constructo implícito y luego será reificado - como la res pública, la reificación pública, ni más, ni menos - y adquiere una evidente identidad simbólica que poco a poco se separa de la práctica, como una declaración imaginada acerca de la práctica. La función ideológica se extiende hasta un punto donde tanto los conservadores como los radicales creen que su práctica va dirigida contra el estado y no contra el contrincante. La tarea del sociólogo es la de desmitificar, lo que en el presente contexto quiere decir considerar los significados según los cuales el estado no existe, más que los significados de acuerdo a los cuales el estado sí existe" (Abrams, 1988:58).

En otro contexto he mencionado que, con estas complicaciones, la tarea de describir el Estado, y todavía peor, explicarlo o por lo menos entenderlo, sería como la burla impagable que nos ofrece mi paisano Sören Kierkegaard en su tesis doctoral de 1843, donde señala que la tarea de observar y describir la ironía en la obra de Sócrates sería como hacer un dibujo de un duende con el sombrero que lo hace invisible.

Pues bien, tenemos que acercarnos a las condiciones para llevar a cabo un estudio etnográfico del Estado. Antes de avanzar más en mi argumento, deseo echar un vistazo a la antropología social británica, que ha logrado sistematizar el trabajo de campo y ha desarrollado un método etnográfico. En la tradición británica hay diversos enfoques, de los cuales quisiera distinguir dos. Por un lado, subraya S. F. Nadel que la antropología sirve para entender las culturas "primitivas", mientras que no nos sirve para entender "(...) nuestra propia civilización, "que conocemos un millón de veces mejor (...) y de la que tenemos datos abundantes y adecuados" (Nadel, 1955:12). Por otro lado, tenemos la antropología de Mary Douglas, de quien leemos que "(...) lo que tiene Douglas de verdaderamente radical es que aplica el mismo diagnóstico para nosotros que para ellos" (MacDonald, 2000:144).

Podemos distinguir un modelo teórico de la Escuela de Manchester, la sociedad plural, y con igual nitidez podemos distinguir tres modelos metodológicos: el análisis situacional, el análisis del caso extendido y el drama social.

La idea de la sociedad plural muestra con claridad que la Escuela de Manchester nació al calor del nuevo orden mundial que surgió sobre las ruinas de la Segunda Guerra Mundial, del cual un elemento fundamental fue el proceso de descolonización: mientras que el objeto de estudio de la antropología había sido tradicionalmente la pequeña comunidad, ahora el objeto de estudio sería la sociedad con sus comunidades. La presentación más precisa de este modelo teórico la encontramos en un largo artículo con el título "Análisis de una situación social en el país zulú moderno" que fue publicado originalmente en 1940, como la parte teórica de otro artículo de Max Gluckman, dedicado a "El reino zulú de Sudáfrica", en el cual Gluckman declara que: 
“(...) he insistido en que desde 1824 ha existido en la tierra zulú una comunidad compuesta por dos grupos culturales, y he sido capaz de aislar comparativamente la organización de la nación zulú ya que sus relaciones con los blancos estaban determinadas por los procesos que ya he descrito. Los eventos fuera del sistema social zulú, en el sistema social de Europa, llevaron al cruce de los dos, creando un nuevo campo de relaciones entre negros y blancos que engendraron nuevas formas de conflicto y cooperación. El cruce inicial, en ocasión y modo, de los dos sistemas era parcialmente gobernado por el azar, aunque esto era en última instancia inevitable. Los desarrollos sucesivos necesariamente estaban determinados por estímulos subyacentes de cada sistema y por los procesos sociales universales" (1958: 36).

Aquí se plantea con precisión que estudiamos un proceso y no una estructura, que nuestro interés se dirige hacia la interacción de las diferentes partes de la sociedad plural con la sociedad global, y que cada parte de la sociedad tiene una autonomía limitada, que en su turno se refleja en la dinámica de la sociedad global. Vale la pena notar dos detalles más: que hacemos uso del concepto de cultura y que prestamos atención a la dimensión histórica de la sociedad, a diferencia de la ortodoxia en la antropología social británica, lo que Max Gluckman elabora en un artículo posterior: "este nuevo tipo de análisis trata cada caso como una etapa en un continuo proceso de relaciones sociales entre personas y grupos específicos en un sistema social y una cultura" (1967: XIII), con énfasis en la palabra proceso.

Con eso nos deslizamos al contenido metodológico del mismo artículo, el análisis situacional, que es presentado así:

“(...) he presentado un ejemplo típico de mis datos del campo. Consiste en un número de eventos que eran articulados por mi presencia como observador, pero ocurridos en diferentes partes del norte del país zulú e involucrando a diferentes grupos de gentes. A través de estas situaciones, y contrastándolas con otras situacio- nes no descritas, intentaré describir la estructura social del país zulú moderno. Las llamo situaciones sociales ya que las analizo en su relación con otras situaciones en el sistema social del país zulú" (Gluckman, 1958: 10).

Se nota una diferencia más, a diferencia de la ortodoxia positivista británica, donde la presencia del investigador o se omite o se relega a una introducción que tiene carácter de confesión, se admite abiertamente como parte de la investigación.

\section{Conclusiones}

Pienso que lo anterior apunta hacia un nuevo proyecto de investigación, emocionante y con muchas perspectivas. Es evidente que la región mesoamericana comparte muchos rasgos con la región andina, pero hay poca claridad en la respuesta a la pregunta: ¿cómo es exactamente la relación entre las dos regiones?, ¿cuál es la dialéctica entre las similitudes y las diferencias, vista a través de la lógica de un proceso histórico, distinguiendo cuidadosamente la larga, mediana y corta duración?

Quisiera mencionar una curiosa asimetría: en Mesoamérica existe, por lo menos a partir de 1937, abundancia de estudios del sistema de cargos; es claro que una institución idéntica al sistema de cargos existe en la región andina, pero apenas contamos con estudios de ella. El libro Introducción al sistema de cargos publicado en 1996, ofrecía una bibliografía del sistema de cargos que en aquel entonces era razonablemente exhaustiva, pero recuerdo que se incluyó solamente un título, el de Harry Tschopik (1947). Estoy plenamente consciente de que existen más títulos hoy, pero de ninguna manera llegan a la altura del caso en Mesoamérica. En la región andina - más precisamente en el Perú - existe una relativa riqueza 
de estudios de la ronda campesina, tal como se desprende de la bibliografía del presente artículo, mientras que los estudios de las instituciones equivalentes a la ronda campesina, las instituciones de defensa de la comunidad, apenas están empezando a surgir (de los pocos ejemplos se puede mencionar el dossier que edité en la revista Pacarina del Sur en el año 2014). La escasez de estudios de las instituciones de defensa de la comunidad se puede deber al cambio reciente en la situación política y socioeconómica en los países de Mesoamérica -México, Guatemala y Belice, principalmente - o bien se puede deber a un cambio en la atención de los antropólogos (recordando que los estudios del sistema de cargos no existían antes del artículo inmortal de Sol Tax en 1937).

\section{Notas}

\footnotetext{
${ }^{1}$ Información verbal de Linda Olvera Farfán, bachiller en antropología social de la Universidad Nacional San Antonio Abad del Cusco, que ha hecho trabajo de campo en esa zona.

${ }^{2}$ En Sol Tax, 1937. Romero y Castaños (1997) llamaron la atención sobre el hecho de que el antropólogo mexicano Noriega ya había descrito y analizado esta institución unos años antes, de una manera que se puede clasificar como el "típico sistema de cargos" (Noriega, 1922). Sin embargo, el trabajo de Noriega fue totalmente olvidado y no dejó huellas en la tradición antropológica de estudio del sistema de cargos, así que el artículo de Sol Tax de 1937 sigue siendo el inicio de esta tradición.

${ }^{3}$ Este típico sistema de cargos ha sido presentado en varias ocasiones. Ver Leif Korsbaek, 1987, 1991. Algunos antropólogos piensan que los dos conceptos, "el típico sistema de cargos" y "el paradigma del sistema de cargos" son construcciones mías; es demasiado honor, la única cosa que hice fue estudiar la existente literatura etnográfica y antropológica y resumir el consenso ampliamente compartido en aquel momento entre los antropólogos en México y Guatemala (en Mesoamérica, si así se prefiere) acerca de la forma y la función del sistema de cargos.

${ }^{4}$ El concepto de "levelling mechanism" ("mecanismo nivelador") fue desarrollado en dos significativos trabajos de Eric Wolf y Manning Nash, ambos publicados en1966.

${ }^{5}$ Nos confirma el sentimiento anticomunista un vistazo a la lista de presidentes de la American Anthropological Association: en 1950, fue presidente Ralph L. Beals; en 1959, Sol Tax; en 1966, John P. Gillin y en 1970 George M. Foster. Esto al mismo tiempo confirma
}

Regresando a la ronda campesina, de nuevo surge el nombre de la abogada peruana Raquel Yrigoyen, "(...) cuando acudió, junto con Ydelso Hernández, a la firma, por parte de la Doctora Silva Chávez, Directora del Centro de Asuntos Interculturales del Ministerio Público (CAIMP) del acto de la CUNARC-PERÜ, de las conclusiones arribadas en el encuentro entre la jurisdicción indígena y la ordinaria hacia un pluralismo jurídico igualitario", el 6 de diciembre del 2016 (Yrigoyen, 2016).

Y volviendo al problema de las duraciones en un proyecto de comparación de las regiones de Mesoamérica y Andina, tenemos que tomar en cuenta la pertenencia del derecho indígena a la larga duración, la historia colonial a la mediana duración y las modificaciones de las jurisdicciones estatales a la corta duración.- la relación entre la AAA y la importancia del estudio del sistema de cargos. R. N. Adams publica un libro más allá de la antropología, con una interpretación también anticomunista del cambio social en América Latina, con artículos del mismo Adams, John L. Gillin y Charles Wagley, entre otros (Adams, 1965).

${ }^{6}$ El volumen cuenta con una introducción y conclusión de Adams y artículos de Robert Ewald (San Antonio Sacatepéquez, 19321953), Kalman H. Silvert \& Arden R. King (Cobán, 1944-1953), John P. Gillin (San Carlos Xilkotepec), Melvin Tumin (también San Carlos Xilotepec), Ruben Reina (Chinautla), Morris Siegel (San Miguel Acatán, 1938-1953), Raymond L. Scheele (Santo Domingo Xenacoj, 1944-1951) y Raymond G. Amir (Magdalena Milpas Altas, 1880-1952). De las fechas de término de los estudios se desprende el interés en el intermezzo comunista en Guatemala, con Arévalo y Arbenz, de 1944 a 1954, y los estudios de Silvert \& King y Scheele empiezan exactamente en 1944.

${ }^{7}$ Gillin (1951) aborda "la cultura de seguridad" en San Carlos, pseudónimo del pueblo San Luis Jilotepec, donde trabajó también Melvin Tumin (1952), quien estudió la estratificación y la movilidad social. La monografía de Mendelson (1965) de Santiago Atitlán es una hermosura.

${ }^{8}$ Según Cancian, la evidencia en Zinacantán claramente indica que "existe algún grado de estratificación económica, a pesar de su operación" (1989: 137-138), y "se encuentra una considerable evidencia de una diferenciación económica dentro de las comunidades en cuestión" (Cancian, 1967: 291). 


\section{Referencias bibliográficas}

Abrams, P. (1988). "Notes on the Difficulty of Studying the State". Journal of Historical Sociology, Vol. 1, № 1, marzo, pp. 58-89.

Adams, R. N. (Ed.). (1957). Political Change in Guatemalan Indian Communities, New Orleans: Tulane University, Middle American Research Institute, Publ. 24.

(1965). Crucifixion by Power. Essays on Guatemalan National Social Structure, 1944-1966, Austin: University of Texas Press.

Barquín Cendejas, A. (2015). Antropología y poder político. El ejercicio de poder en las políticas de educación intercultural, México: Instituto Nacional de Antropología e Historia.

Bauman, Z. (1972). "Tiempos modernos, marxismo moderno". En Berger,P (comp.) Marxismo y sociología, Buenos Aires: Amorrortu, pp.17-31.

Bourdieu, P., Wacquant, L.D. \& Farage, S. (1994). "Rethinking the State: Genesis and Structure of the Bureaucratic Field". Sociological Theory, Vol. 12, № 1: 1-18.

Cámara Barbachano, F. (1952). "Religious and Political organization". En Tax, S. (ed.) "Heritage of Conquest". The Free Press, Glencoe, III., 1952: 142-173 (publicado en español en Korsbaek, L. (ed.) (1996). "Introducción al sistema de cargos", Facultad de Antropología de la UAEM, Toluca, pp. 113-159).

Cancian, F. (1967). "Political and Religious Organization". In Handbook of Middle American Indians, Austin: University of Texas Press, Vol. 6: 283-298.

(1989). Economía y prestigio en una comunidad maya.

México: CNCA/INI.

Carrasco, P. (1961). "La jerarquía cívicorreligiosa en las comunidades de Mesoamérica: antecedentes precolombinos y desarrollo colonial". En Llobera, J.R. (comp.). (1979) Antropología Política, Barcelona: Anagrama, pp. 323-340.

Castaingts, J. (1977). La articulación de los modos de producción. México D.F.: El Caballito.

Castañeda García, I. (s. f.). "Comités de autodefensa en el periodo post violencia política”. Disponible en: http://www.resdal.org/ producciones-miembros/art-castaneda06.html Fecha de consulta: 26 de junio de 2016

Chance, J. K. \& Taylor, W.B. (1985). "Cofradías y cargos: Una perspectiva histórica de la jerarquía cívico-religiosa mesoamericana”. Antropología. Suplemento № 14, INAH, México.

Coser, L. (1961). Las funciones del conflicto social. México: Fondo de Cultura Económica.

Defensoría del Pueblo (2004). El reconocimiento estatal de las rondas campesinas. Compendio de normas y jurisprudencia. Lima, Perú.

Degregori, C. I., Coronel, J. \& Starn, O. (1990). La derrota de sendero luminoso. Lima: Instituto de Estudios Peruanos.
Degregori, C.I. \& Ponce Mariños, M. (2000). "Movimientos sociales y estado. El caso de las rondas campesinas de Cajamarca y Piura". En Degregori, C.I. (ed.). No hay país más diverso. Compendio de antropología peruana. Lima: IEP/PUCP/Universidad del Pacífico, pp. 392-412.

Diener, P. (1978). "The tears of Saint Anthony: Ritual and Revolution in Eastern Guatemala”. Latin American Perspectives, Vol. 18: 92-116.

Gillin, J. P. (1951). The Culture of Security in San Carlos. A Study of a Guatemalan Community of Indians and Ladinos. New Orleans: Tulane University (Middle American Research Institute, Publ. № 16).

Gluckman, M. (1958). "Analysis of a Social Situation in Modern Zululand", Manchester: University of Manchester Press. (1967). "Introduction". In Epstein, A. L.(ed.). The Craft of Social Anthropology, London: Tavistock, XI-XX.

(1972). "Moral Crisis: Magical and Secular Solutions". In Gluckman, M. (ed). The Allocation of Responsibility. Manchester University Press, 1972: 1-50.

Greenberg, J. B. (1987). Economía y religión entre los chatinos. México: Instituto Nacional Indigenista.

Huber, L. \& J. C. Guerrero (2006). "Las rondas campesinas de Chota y San Marcos", Lima: PROJUR/SER.

Huber, L. (1995). "Después de Dios y la Virgen está la ronda. Las rondas campesinas de Piura”, IEP/IFEA, Colección Mínima №31.

Huerta Ríos, C. (1997). "Análisis genético-funcional del sistema de cargos en una etnia en transformación". 1er Coloquio del Sistema de Cargos, Toluca, abril.

Korsbaek, L. (1987). "El desarrollo del sistema de cargos de San Juan Chamula: El modelo teórico de Gonzalo Aguirre Beltrán y los datos empíricos". Anales de Antropología Vol. 24: 215-242.

(1991). "La religión y la política en el sistema de cargos: Una comparación de tres comunidades mayas en los Altos de Chiapas". Cuicuilco, № 23-24: 115-132.

(ed.) (1996). Introducción al sistema de cargos, Toluca: UAEM.

(2009a). La etnografía de una comunidad matlatzinca en el Estado de México: el sistema de cargos y la neoetnicidad en San Francisco Oxtotilpan, Municipio de Temascaltepec (Tesis Doctoral en Ciencias Antropológicas), Universidad Autónoma Metropolitana Iztapalapa.

(2009b). "Instituciones de defensa de la comunidad". Revista Ra Ximhai, Vol. 5, № 3, sep-dic. pp. 373-385.

(2012). "El cambio de las instituciones comunitarias bajo el impacto del neoliberalismo". Ponencia presentada en el $53^{\circ}$ Congreso Internacional de los Americanistas, Viena.

(ed.) (2014). Dossier № 12: “Instituciones de defensa de la comunidad". Pacarina del Sur, julio. 
(2016). "Los estudios políticos en la Escuela de Manchester". Alma Mater Universidad de San Marcos, Lima, Vol. 3, № 4, mayo, pp. 75-88.

Kuhn, T. S. (2007). La estructura de las revoluciones científicas, México: Fondo de Cultura Económica.

LaFarge, O. \& Byers, D. (1931). The Year Bearers' People. New York: Middle American Research Series, Publication Number 3, Department of Middle American Research, Tulane University.

Laos Fernández, A., P. Paredes \& Rodríguez, E. (2003). Rondando por nuestra ley. Lima: RID/SER.

Lerner, S. (2003). Informe final de la Comisión de Verdad y Reconciliación. Lima: Presidencia de la República.

Macdonald, T. (2000). "Sociedades plurales". En Barfield, T. (ed.). Diccionario de Antropología. México: Siglo XXI, pp. 490-491.

Machaca, A. (2000). "Rondas campesinas". Runa. Ayaviri: Vicaría de Solidaridad de Ayaviri, № 43.

Malinowski, B. (2015). "El aspecto económico de la ceremonia de Intichiuma" (Traducción de Leif Korsbaek). En Boletín de Antropología, Universidad de Antioquia, Medellín, Vol. 29, № 47, primer semestre, pp. 213-232.

Mendelson, E. M. (1965). Los escándalos de Maximón. Guatemala: Tipografía Nacional de Guatemala.

Nadel, S. F. (1955). The Foundations of Social Anthropology, London, Cohen \& West.

Nash, M. (1966). Primitive and Peasant Economic Systems, Chandler, San Francisco, 1966.

(1996). "Relaciones Políticas en Guatemala". En Korsbaek,

L. (ed.) Introducción al sistema de cargos". Toluca: Facultad de Antropología de la UAEM, pp. 161-174. (Trabajo original publicado en 1958).

Noriega Hope, C. (1979). "Etnografía”. En Gamio, M. (comp.) La población del Valle de Teotihuacan, Tomo IV, México D.F.: Instituto Nacional Indigenista, pp. 203-281.

Paul, B. D. (1976). "Mental Disorder and Self-Regulating Processes in Culture. A Guatemalan Illustration". In Hunt, R. (ed.) Personalities and Cultures. Readings in Psychological Anthropology. Austin: University of Texas, pp. 150-165.

Pérez Mundaca, J. (1994). "Poder, violencia y campesinado en Cajamarca. El caso de la Microrregión Central”. En Perú: El problema agrario en debate, SEPIA IV, pp. 459-485.

Price, D. H. (2004). Threatening Anthropology. McCarthyism and the FBI's Surveillance of Activist Anthropologists, Durham \& London: Duke University Press.

Ramírez Salazar, R. H. (2007). Justicia ordinaria y justicia comunal en Andahuaylas-Apurímac. Una mirada a las instituciones locales de la administración de justicia. Lima: PROJUR.

Ribeiro, D. (1977). Las Américas y la civilización, México: Editorial Extemporáneos.

Rodríguez Aguilar, C. (2007). "Las rondas campesinas en el sur andino", Lima: PROJUR/SER.
Rojas, T. (1990). "Rondas, poder y terror". Alternativa, Revista de Análisis del Norte, № 13, mayo, pp. 83-120.

Romero Contreras, A. T. (1997). "La primera descripción del sistema de cargos en la etnografía mexicana". Ponencia presentada en Korsbaek, L. $1^{\text {er }}$ Coloquio del Sistema de Cargos, Toluca: México.

Siverts, H. (1964). "On Politics and Leadership in Highland Chiapas". En Vogt, E. Z.\& Rus, A. L. (eds.) Desarrollo Cultural de los Mayas, México D.F.: UNAM, pp. 363-384.

(1965a). "Some Economic Implications of Plural Society".

FOLK. Vol. 7, pp. 153-162.

(1965b). "The Cacique of Kancuk: A Study of Leadership in Highland Chiapas", Estudios de Cultura Maya, México: UNAM, Vol. V, pp.339-360.

(1969). Oxchuk: Una tribu maya de México, México D.F.: Instituto Indigenista Interamericano, Ediciones Especiales № 52.

Smith, W. R. (1981). "El sistema de fiestas y el cambio socioeconómico", México: Fondo de Cultura Económica.

Starn, O. (1991). "Con los llanques todo barro. Reflexiones sobre rondas campesinas, protesta rural y nuevos movimientos sociales", Lima: IEP.

Tax, S. (1937). "The Municipios of the Midwestern Highlands of Guatemala". American Anthropologist Vol.39: 423-444 (publicado en español en Korsbaek, L. (comp.) Introducción al sistema de cargos. Toluca: Facultad de Antropología de la UAEM: 87-112) (Trabajo original publicado en 1937).

Tschopik, H. (1947). "Highland Communities of Central Peru". Washington D.C.: Smithsonian Institution, Publ. № 5.

Tumin, M. (1952). Caste in a Peasant Society: A Case Study in the Dynamics of Caste, Princeton: University of Princeton Press.

Wasserstrom, R. (1977). Red Fathers, White Souls: Indian-Ladino Relations in Highland Chiapas, 1528-1973, (Doctoral Dissertation) Massachussets: Harvard.

Wolf, E. (1966). The Peasants. Englewood Cliffs, New Jersey, Prentice Hall.

Yrigoyen, R. (2003). "Pluralismo jurídico, derecho indígena y jurisdicción especial en los países andinos". Ponencia presentada en el foro internacional Pluralismo jurídico y jurisdicción especial, febrero, Lima.

(2016). "Acuerdos en el encuentro entre la jurisdicción indígena y la ordinaria: Hacia un pluralismo jurídico igualitario". Firmados por la Dra. Leticia Silva Chávez, Directora del Centro de Asuntos Interculturales del Ministerio Público (CAIMP) y CUNARC, Lima, PUCP, 6 de diciembre.

Zarzar, A. (1991). "Las rondas campesinas de Cajamarca: de la autodefensa al ¿autogobierno?”. Pásara, L., Valdeavellano, R. \& Zarzar, A. (eds.). La otra cara de la luna. Nuevos actores sociales en el Perú. Buenos Aires: CEDYS. 
